\title{
Relaciones entre población, desarrollo y pobreza según los profesores universitarios mexicanos
}

\section{Óscar Cuéllar S.*}

Con base en los datos de una encuesta aplicada a muestras aleatorias de profesores universitarios de siete ciudades de México en la segunda mitad de los años ochenta, el artículo examina las opiniones de los maestros acerca de las relaciones entre población, desarrollo y pobreza. Luego de reseñar las tendencias y argumentos en la literatura sobre población y desarrollo, ofrece tres tipologías simples de la conciencia poblacional, los determinantes de la pobreza y el tipo de desarrollo económico deseable para el país. A continuación estudia las relaciones entre ellas y concluye esbozando el perfil argumentativo de las principales orientaciones detectadas por el análisis.

\section{Introducción}

Este trabajo tiene como objetivo examinar algunos aspectos de la problemática de las relaciones entre población y desarrollo, con base en los datos de una encuesta aplicada en la segunda mitad de los años ochenta a una muestra de maestros universitarios mexicanos de provincia. ${ }^{1}$

Se trata de ofrecer un panorama de las orientaciones de los maestros universitarios sobre el tema, tomando como base algunos puntos centrales de la discusión habida: $a$ ) la importancia que se atribuye al tamaño y crecimiento de la población vis-à-vis el desarrollo; b) las vinculaciones que se establecen entre el crecimiento poblacional y la explicación de la pobreza. Además, estudiamos

* Departamento de Sociología, Universidad Autónoma Metropolitana, Unidad Azcapotzalco.

1 En este trabajo se usan los datos de la encuesta a profesores universitarios de provincia del proyecto MEX/87/P01, del Fondo de Población de las Naciones Unidas (convenio con la Universidad Iberoamericana). La encuesta se aplicó en siete ciudades de la República: León, Guanajuato; Torreón, Coahuila; Puebla, Puebla; Guadalajara, Jalisco; Hermosillo, Sonora, y Tijuana y Mexicali, Baja California. En Mexicali la encuesta se aplicó en enero de 1988 y en las demás, en el segundo semestre de 1987. Con base en la información de la Asociación Nacional de Universidades e Instituciones de Enseñanza Superior (ANuies, 1986), se seleccionó una muestra aleatoria estratificada de los profesores universitarios en etapas: $a$ ) de universidades, tomando en cuenta la distribución entre universidades públicas y privadas; $b$ ) de facultades y escuelas, distinguiendo entre ciencias sociales y humanidades, por un lado, y ciencias naturales, por otro, y c) de horarios (mañana, tarde, noche). Se seleccionaron aleatoriamente los horarios de las escuelas y se entrevistaron a los maestros al inicio o término de la clase. El tamaño muestral fue de entre 
sus opiniones sobre el tipo de desarrollo deseable y el papel que otorgan a la incorporación de tecnología frente al empleo de la fuerza de trabajo en las estrategias de desarrollo. El trabajo se ordena de la siguiente manera: primero, resumimos algunos de los puntos centrales de la discusión sobre población y desarrollo que se dio en México sobre todo en la década de los setenta, pero que continúan siendo tema de discusión entre los analistas. A continuación analizamos los datos de la encuesta y presentamos los resultados; por último, bosquejamos el perfil argumentativo de las principales orientaciones detectadas, teniendo como referente las polémicas previas sobre el tema.

\section{Población y desarrollo: un panorama general}

La problemática de las vinculaciones entre la población y el desarrollo ha sido objeto de preocupación por parte de académicos y políticos desde hace ya varios lustros (Eversley, 1959). Los orígenes del moderno interés por el tema se pueden situar en el periodo inmediatamente posterior a la Segunda Guerra Mundial, cuando la información censal mostró que, contra las predicciones previas, la población estaba creciendo a un ritmo acelerado, especialmente en los países subdesarrollados -lo que fue corroborado por los censos de los años setenta (CEPAL, 1983; Carrillo Flores, 1974).

La pregunta central que se plantearon inicialmente los estudiosos se refirió a los efectos que podría tener el crecimiento demográfico en el futuro económico y en el bienestar de los países de menor desarrollo (un trabajo clásico en esta línea fue el de Coale y Hoover, 1958). Bajo el supuesto de que aumentos excesivos

160 y 200 casos, salvo en Mexicali (105). En León, Guadalajara, Puebla y Torreón alcanzó el número máximo, mientras que en Hermosillo fue de 160 casos y en Tijuana, de 176 (sobre los procedimientos de muestreo y determinación del tamaño de muestra, véase Cortés, 1982). Se aplicó un cuestionario con preguntas cerradas y semicerradas estructurado en módulos: antecedentes personales, trayectoria educativa y laboral; percepciones de la problemática poblacional, educativa y laboral; percepciones de la problemática poblacional, y conocimientos, opiniones y evaluaciones acerca de la problemática "población-desarrollo". Las entrevistas se realizaron con el apoyo de profesores y estudiantes universitarios de cada ciudad.

Del número total de instituciones de educación superior existente en los estados a que pertenecen las ciudades, más de la mitad se ubica en éstas. Algo similar sucede con los maestros. La única excepción es Torreón, que comprendía $42 \%$ de las instituciones y $44 \%$ de los maestros universitarios del estado de Coahuila, a la fecha de la encuesta. 
en la población podrían significar una fuerte demanda de inversiones no productivas que en algún momento llevarían a una disminución o a un estancamiento del crecimiento económico, proliferaron los trabajos que examinaron los efectos que las tasas de crecimiento poblacional de los países subdesarrollados tenían o podrían tener sobre aspectos tales como la capacidad de ahorro (de países y familias), la inversión, la productividad, la igualdad económica ("o disparidad") y la pobreza. En otros casos, se trabajó con sofisticados modelos matemáticos que, además de los factores poblacionales, incorporaron variables relativas al ambiente, arrojando sombríos pronósticos sobre el futuro (Meadows et al., 1972; Mesarovic y Pestel, 1974).

Como resultado de ello y a pesar de que en la región el crecimiento poblacional en el periodo de posguerra había sido acompañado por índices relativamente altos de desarrollo (Petch, 1979; Fucaraccio, 1979), desde fines de los años sesenta se difundió una importante campaña para sensibilizar a la población sobre los peligros que podrían derivarse del crecimiento demográfico acelerado. ${ }^{2}$ Los organismos internacionales y las agencias no gubernamentales privadas presionaron a los gobiernos para que diseñaran y aplicaran políticas dirigidas a inducir o regular el comportamiento demográfico de la población a fin de hacer posible el crecimiento económico.

En el caso mexicano, estas campañas encontraron un medio intelectual y político favorable que llevó, a principios de los años setenta, a un cambio en las orientaciones del gobierno en la materia (Carrillo Flores, 1974). De una posición claramente "pro-natalista", se pasó a un esfuerzo por disminuir la fecundidad, señalada como responsable del acelerado crecimiento poblacional. Al mismo tiempo, el gobierno planteó que la política poblacional debía darse en el contexto del respeto por los derechos de las familias y de las libertades consagradas por la constitución (United Nations, 1989a; Alba y Potter, 1986).

Como han apuntado Rodríguez y Yocelevzky (1986), tal vez el hecho de que la problemática demográfica apareciera en la forma de una promoción de las políticas de control natal dio base a la álgida confrontación que tuvo lugar. En particular en el campo académico, la polémica en torno de la cuestión poblacional en América Latina llevó a fuertes debates, derivados de las distintas afiliaciones

2 Según Urquidi y Morelos (1979: 3-8), "los principales señalamientos sobre las consecuencias de la dinámica de la población se pronunciaron durante la década de los setenta". Para una visión crítica, véase Astorga Almanza (1987). 
ideológicas y científicas con que se asociaron las posiciones en juego. En términos generales, éstas se polarizaron de tal forma que mientras, por un lado, los abogados de la necesidad de una mayor conciencia poblacional aparecían estrechamente ligados a las orientaciones teóricas neomalthusianas, por otro, católicos y marxistas, coincidían en el rechazo de esas posiciones, aunque por motivos diferentes (Leñero, 1974; Coale, 1982). ${ }^{3}$

Sin embargo, hacia la segunda mitad de la década siguiente los ánimos llegaron a permitir una mirada menos apasionada de las cuestiones poblacionales. El punto de inflexión se inició con la Conferencia Mundial de Población (Bucarest, 1974), convocada para discutir específicamente "los problemas demográficos básicos, sus relaciones con el desarrollo económico y social y las políticas de población y los programas de acción necesarios para promover el bienestar humano y el desarrollo" (United Nations Secretariat, 1987: 76). Los foros internacionales legitimaron la idea de que la política poblacional debía ponerse al servicio del bienestar y el desarrollo de los pueblos, lo que exigiría su integración en los planes de desarrollo (Miró y Potter, 1980; Cabrera, 1984; González, 1984; United Nations Secretariat, 1987). Así, desde los años ochenta, la problemática "población-desarrollo" ha adquirido carta de ciudadanía en la discusión científica, que se ha preocupado por establecer el ámbito que permita una indagación más serena de los temas cruciales, alejada del calor de las tomas de posición anteriores.

En gran parte, el cambio en la agenda fue resultado de los avances en la investigación y de la discusión producto de ella. Entre otras cosas, se criticó la asociación simplista entre crecimiento demográfico y productividad o ingreso per cápita (Rodgers, 1983; Mason, 1987); se indicó que las mediciones a partir de pocas variables a niveles agregados a escala mundial son poco confiables (Anker y Farooq, 1978), y se señaló que un problema teórico de base de los modelos económicos y las proyecciones para países menos desarrollados consistió en que se asentaban en supuestos carentes de fundamento científico. ${ }^{4}$

${ }^{3}$ En la región el incipiente marxismo de fines de los años sesenta, generalmente ligado a posiciones nacionalistas o antimperialistas en lo ideológico, se asoció con la idea del socialismo como vía para el desarrollo (Rodríguez y Yocelevzky, 1986) y la acusación de que plantearse el problema poblacional no era más que una manera de disfrazar la preferencia por la conservación del status quo, y de evitar todo intento de disminuir la concentración de la riqueza. Así, ésta aparecía como la verdadera causa de la pobreza y del problema poblacional.

4 Samuel H. Preston (1987: 1-10) ha puesto la cuestión de modo irónico: "In 
También en México las posiciones se han matizado (comparar, por ejemplo, Benítez Zenteno, 1965 y 1989), y la idea de que es menester persuadir a la población de los negativos efectos derivados de un crecimiento rápido ha ganado aceptación generalizada. Este planteamiento no podría calificarse de antojadizo. Si bien es cierto que las tasas de crecimiento demográfico muestran síntomas claros de declinar (United Nations, 1989a; 1989b), la magnitud global de la población ya alcanzada asegura que el incremento en el número de habitantes seguirá constituyendo un problema para las estrategias del desarrollo, agravado por los efectos de la crisis económica de principios de esta década y, en especial, por las políticas de ajuste y cambio estructural que le sucedieron (Cortés y Rubalcava, 1991). Tiene entonces sentido preguntarse por las posiciones en juego y, en particular, por las variaciones que puedan exhibir en la actualidad. Es lo que haremos a continuación, tomando como referente a los profesores universitarios de provincia.

\section{Población, pobreza y desarrollo}

Para analizar las orientaciones de los profesores universitarios en materia de población y desarrollo, seleccionamos tres temas básicos, que trabajamos en la forma de tipologías simples: a) la conciencia poblacional; b) los determinantes de la pobreza y $c$ ) tipo de desarrollo deseable para el país.

En primer lugar, se ofrece una sistematización del tipo de "conciencia poblacional". Este término se refiere a las percepciones acerca del significado que tiene el volumen actual de la población del país respecto del crecimiento económico, y se construyó empleando las respuestas a dos preguntas: i) "en México hay exceso de población" y ii) "el crecimiento de la población es un problema para el

matters of human being it is thought that per capita measures are what really matter. But accepting them as the definitive indicators of human welfare inmmediately creates a link measures of well-being appear to give population size coequal importance with whatever appears in the numerator. With any given numerator (goods), an increase in the denominator (population growth) spreads the goods more thinly and results in a loss of well-being. The logic of the connection is unassailable. Since many of the goods involve products of nature-land, minerals, energy, species-and since the abundance of nature is seen as more or less fixed, it becomes reasonable to think that population growth diminishes the per capita availability of most goods. It seems fair top say that this is the predominant way in which the issue of economic effects of population growth has traditionally been considered. It is an approach that can be traced back at least to Malthus". 
desarrollo del país". ${ }^{5}$ La clasificación pone, por un lado, a los que parecen mostrar un seria preocupación por el tamaño y crecimiento de la población y, por el otro, a los que no ven problemas en estos aspectos o no parecen tener una idea estructurada acerca de su posible significado.

Denominamos "alertas" a quienes consideran que hay exceso de población y que su crecimiento es un problema para el desarrollo del país; "preocupados", a los que no creen que haya exceso de población, o piensan que la afirmación es dudosa, pero ven en el crecimiento demográfico un problema para el futuro. Y "optimistas" a los que no piensan que haya exceso de población ni que su crecimiento represente problemas. Esta categoría comprende también los casos en los cuales no se ve problema en el crecimiento y se considera dudosa la afirmación según la cual hay exceso de población en el país.

Al resto (los inconsistentes, que piensan que hay exceso, pero no ven problemas en el crecimiento de la población, y los que consideran que ambas afirmaciones son dudosas) los incluimos en la categoría "desapercibidos".

Las percepciones sobre los "determinantes de la pobreza" toman en cuenta las opiniones acerca del papel del crecimiento demográfico y de la concentración de la riqueza en la explicación de la pobreza (Argüello, 1979). La tipología permite tener una idea del grado en que se ve esta última como una consecuencia del crecimiento poblacional (recuérdese que éste ha sido un argumento frecuentemente esgrimido en las controversias sobre la "explosión demográfica" y que ha sido combatido por las tendencias más radicales, que ponen el acento en los mecanismos derivados de la concentración de la riqueza). Se construyó con las respuestas a las secciones: i) "el crecimiento excesivo de la población es la causa principal de la pobreza" y ii) "la causa principal de la pobreza es la concentración de la riqueza".

Llamamos "eclécticos" o moderados a quienes consideran que en la explicación de la pobreza deben incluirse tanto aspectos demográficos como económico-sociales. "Malthusianos" fueron considerados los que pusieron el acento en el papel de los factores demográficos en desmedro de los económico-sociales. A quienes, por el contrario, enfatizaron estos últimos, negando los

5 Las secciones de la encuesta que se emplearon para construir las tipologías comprendían cinco alternativas de respuesta. Éstas se recodificaron reuniendo "acuerdo" y "completo acuerdo" por una parte, y "desacuerdo" y "completo desacuerdo", por otra. Se mantuvo la respuesta "depende", y no se consideraron las no respuestas en el análisis. 
determinantes demográficos o atribuyéndoles un papel secundario, los denominamos "radicales". La categoría "otros" comprende las demás respuestas.

Las opiniones acerca de la clase de desarrollo económico deseable para el país llevaron al tercer tipo, que se elaboró con las respuestas a las secciones: i) "el desarrollo económico del país debería basarse en el uso intensivo de la fuerza de trabajo" y ii) "el crecimiento económico del país debería fundamentarse en la incorporación de los avances tecnológicos".

En la literatura sobre el tema, esta dimensión no ha llevado a argumentaciones enconadas en las discusiones serias que han tenido lugar. Sería difícil encontrar posiciones extremas en esta materia ya que, si bien la preocupación por la existencia de excedentes de fuerza de trabajo favorece las inclinaciones por buscarles solución, parece evidente que reposar exclusivamente en ello podría significar que no se puedan alcanzar los objetivos del desarrollo. Un crecimiento centrado en la tecnología, por otro lado, por sí mismo no aliviaría el problema económico, social y político de la sobrepoblación, por lo menos en el corto o mediano plazo.

Por tanto, la mayor posibilidad es esperar (desear) que las estrategias de desarrollo busquen una combinación óptima, equilibrando, en la medida de lo posible, el uso de la fuerza de trabajo disponible con la incorporación de los avances tecnológicos (el tipo de desarrollo deseable debe ser "equilibrado").

De la construcción de la tipología surgen, de todos modos, posiciones más extremas: privilegiar el uso de la tecnología (desarrollo con base en la tecnología); o -lo que parece menos probablede la fuerza de trabajo con desprecio de la tecnología. Las demás respuestas (inconsistentes y dudosas) se incluyeron en "otros".

En síntesis, mientras el primer tema remite a la importancia que se atribuye al crecimiento demográfico, el segundo nos aproxima a la visión acerca del papel que se otorga a los factores demográficos en la generación de la pobreza. En ambos casos, se trata de percepciones acerca de procesos que han dado lugar a debates y discusiones que suelen tener un espacio en el ámbito universitario. El tercero pone más bien la cuestión en el plano de lo deseable, y se sujeta menos que los otros a la influencia de las discusiones habidas. Conviene ver los datos (cuadro 1).

En la parte superior del cuadro se aprecia que poco más de $40 \%$ de los maestros entrevistados coincidieron en que la población del país es excesiva y que su crecimiento representa un problema para el desarrollo nacional ("alertas"). Si se agregan los que coinciden con la última proposición ("preocupados") resulta que alrededor de $70 \%$ parecen tener cierta conciencia de la gravedad 
CUADRO 1

Tipología de orientaciones en materia de población y desarrollo de los maestros universitarios: conciencia poblacional, determinantes de la pobreza y tipo de desarrollo deseable (siete ciudades de México, 1987)

\begin{tabular}{|c|c|c|c|c|}
\hline Orientaciones & VaI & ables & Distribu & ción \\
\hline $\begin{array}{l}\text { Conciencia } \\
\text { poblacional } \\
\text { - Alertas }\end{array}$ & $\begin{array}{l}\text { Exceso de } \\
\text { población } \\
\text { sí }\end{array}$ & $\begin{array}{c}\text { Crecimiento } \\
\text { es problema } \\
\text { sí }\end{array}$ & $\begin{array}{c}\text { Número } \\
1267 \\
567\end{array}$ & $\begin{array}{c}\% \\
100.0 \\
44.8\end{array}$ \\
\hline - Preocupados & $\begin{array}{c}\text { depende } \\
\text { no }\end{array}$ & $\begin{array}{l}\text { sí } \\
\text { sí }\end{array}$ & 337 & 26.6 \\
\hline - Optimistas & $\begin{array}{c}\text { no } \\
\text { depende }\end{array}$ & $\begin{array}{l}\text { no } \\
\text { no }\end{array}$ & 244 & 19.2 \\
\hline - Otros & sí & no & 119 & 9.4 \\
\hline $\begin{array}{l}\text { Determinantes } \\
\text { de la pobreza } \\
\text { - Eclécticos }\end{array}$ & $\begin{array}{c}\text { Crecimiento } \\
\text { población } \\
\text { sí } \\
\text { depende }\end{array}$ & $\begin{array}{c}\text { Concentración } \\
\text { de riqueza } \\
\text { sí } \\
\text { sí }\end{array}$ & $\begin{array}{c}\text { Número } \\
1266 \\
323\end{array}$ & $\begin{array}{c}\% \\
100.0 \\
25.5\end{array}$ \\
\hline - Malthusianos & $\begin{array}{c}\text { sí } \\
\text { sí } \\
\text { depende }\end{array}$ & $\begin{array}{c}\text { no } \\
\text { depende } \\
\text { no }\end{array}$ & 99 & 7.8 \\
\hline - Radicales & $\begin{array}{l}\text { no } \\
\text { no }\end{array}$ & $\begin{array}{c}\text { sí } \\
\text { depende }\end{array}$ & 619 & 48.9 \\
\hline - Otros & $\begin{array}{c}\text { no } \\
\text { depende }\end{array}$ & $\begin{array}{c}\text { no } \\
\text { depende }\end{array}$ & 225 & 17.8 \\
\hline $\begin{array}{l}\text { Tipo desarrollo } \\
\text { deseable } \\
\text { - Equilibrado }\end{array}$ & $\begin{array}{l}\text { Bas } \\
\text { F. trabajo } \\
\text { sí }\end{array}$ & $\begin{array}{l}\text { do en } \\
\text { Tecnolog. } \\
\text { sí }\end{array}$ & $\begin{array}{c}\text { Número } \\
1269 \\
840\end{array}$ & $\begin{array}{c}\% \\
100.0 \\
66.2\end{array}$ \\
\hline - Base, F. trabajo & $\begin{array}{c}\text { sí } \\
\text { sí } \\
\text { depende }\end{array}$ & $\begin{array}{c}\text { no } \\
\text { depende } \\
\text { no }\end{array}$ & 152 & 12.0 \\
\hline - Base, tecnología & $\begin{array}{c}\text { no } \\
\text { depende } \\
\text { no }\end{array}$ & $\begin{array}{c}\text { sí } \\
\text { sí } \\
\text { depende }\end{array}$ & 194 & 15.3 \\
\hline - Otro & no & no & 83 & 6.5 \\
\hline
\end{tabular}

Fuente: Encuesta a maestros universitarios de provincia, Fondo de Población, NU/U. Iberoamericana. 
que pueden representar los aspectos demográficos en el crecimiento económico.

No obstante, no deja de llamar la atención que casi una quinta parte de los maestros no parece creer que haya exceso de población ni que el crecimiento demográfico sea problema.

En lo que se refiere ahora a la explicación de la pobreza, resalta la proporción que ve su causa principal en la concentración de la riqueza ("radicales", 48.9\%). Una cuarta parte de los profesores cree que juegan tanto factores socioeconómicos como demográficos ("eclécticos"), y sólo una pequeña minoría atribuye la pobreza fundamentalmente al crecimiento poblacional. Si se junta a los "eclécticos" con los "malthusianos", ambos constituyen sólo un tercio de los maestros que señala algún factor demográfico en la determinación de la pobreza.

En cuanto al tipo de desarrollo deseable, dos terceras partes de los entrevistados creen que el tipo de desarrollo deseable es aquel que combina el uso de la fuerza de trabajo excedente con la incorporación de tecnología. Un punto de vista más "tecnócrata", que enfatiza sólo la incorporación de tecnología, tiene escasa representación y es apenas poco más importante que su opuesto, que preferiría que el desarrollo se basara en el uso de la fuerza de trabajo y no en la tecnología.

En síntesis, una primera visión de las orientaciones de los maestros indica que éstos tienden a considerar que hay exceso de población o bien que el crecimiento demográfico es un problema para el desarrollo del país; se inclinan, sin embargo, a atribuir la pobreza a factores socioeconómicos más que demográficos y, en todo caso, opinan que el desarrollo deseable sería aquel que combinara el uso de la fuerza de trabajo excedente con la incorporación de los avances tecnológicos.

\section{Crecimiento demográfico y pobreza}

Cabe recordar que uno de los temas más debatidos en torno a la cuestión poblacional se refiere al impacto del crecimiento demográfico sobre el bienestar y, en particular, sobre el ingreso y la igualdad económica entre los individuos. Como indica Rodgers (1983: 363):

Para muchos observadores, el rápido crecimiento de la población frena la extensión de las infraestructuras sociales, reduce el crecimiento de los ingresos por habitante, aumenta la presión sobre los limitados recursos naturales y conduce a excedentes de mano de obra y al desempleo. 
Se ha discutido bastante qué tan certeros son estos temores (Rodgers 1983; Urquidi y Morelos, 1979; Anker y Farooq, 1978; Rodríguez y Yocelevzky, 1986). Aunque la discusión aún no acaba, los ánimos se han morigerado. En efecto: a) en lo que toca a las relaciones entre crecimiento demográfico e ingreso, los resultados de las investigaciones no son concluyentes y algo similar sucede con la relación entre cambio demográfico, ahorro y crecimiento económico (Mason, 1987); b) en lo que se refiere a la desigualdad, parece prevalecer actualmente la opinión de que "los análisis publicados, ya sean nacionales o internacionales, no demuestran de manera convincente que el crecimiento demográfico elierza sobre la disparidad efectos importantes" (Rodgers, 1983: 381). Sin embargo, en algunos casos se sigue sosteniendo (aunque no se haya probado convincentemente) que en la raíz de la crisis económica actual del país, que ha significado aumentos notorios en la disparidad, se encuentra el acelerado crecimiento poblacional de las décadas recientes (véase, por ejemplo, Alba y Potter, 1986).

Siguen, pues, existiendo diferencias sobre el tema. Podría conjeturarse que probablemente la estructura de los argumentos y las confrontaciones referidas no han variado demasiado y, además, que se han hecho presentes también en los medios académicos de la provincia. En efecto, éstos se han visto sometidos no sólo a los esfuerzos académicos por hacer conciencia de la problemática poblacional sino, además, a información continua emitida por los medios masivos de comunicación social, donde se insiste en los probables efectos negativos del crecimiento demográfico (sobre esto, véase Muñiz, 1988; 1989).

Así, sería razonable esperar que hubiese relación positiva entre la conciencia poblacional y el papel atribuido a los factores demográficos en la explicación de la pobreza. Habría que conceder, sin embargo, que esta tesis puede resultar demasiado radical: a la luz de las evidencias actuales, sería difícil negar la posibilidad de que interactúen factores demográficos y socio-económicos, colaborando en la explicación.

Por otra parte, y debido a la vinculación que históricamente se ha dado en América Latina entre radicalismo y argumentos antidemográficos (más precisamente: antinatalistas), podría esperarse que los "optimistas" no atribuyeran la misma importancia a los primeros, mientras que los "desapercibidos" o bien coincidiesen con los optimistas o bien tuviesen respuestas poco consistentes en lo que se refiere a la explicación de la pobreza.

Con el propósito de apreciar las posibles resonancias de tales confrontaciones y examinar de qué manera ven los profesores universitarios las vinculaciones entre crecimiento demográfico y 
pobreza, relacionamos los datos de la tipología de la conciencia poblacional de los maestros con la tipología referida a las explicaciones de la pobreza. La información se presenta en el cuadro 2, en donde agrupamos a los "alertas" y "preocupados", por una parte ("conscientes") y a los "optimistas" y al resto, por otra ("otros").

Resulta clara la relación entre el tipo de conciencia poblacional y el tipo de explicaciones acerca de los determinantes de la pobreza:

a) Los "conscientes" son, o bien radicales ( $42.9 \%)$ en la explicación de la pobreza, o bien "equilibrados" (30.9\%). Entre los conscientes, además, hay una pequeña proporción (16\%) que no podría clasificarse en términos de las categorías empleadas (no recurren a criterios demográficos ni socioeconómicos para explicar la pobreza). Finalmente, muy pocos $(10 \%)$ de los que tienen alguna conciencia poblacional pueden calificarse de "malthusianos", es decir, que explicarían la pobreza recurriendo exclusivamente a argumentos demográficos.

b) Por lo que respecta a los que no tienen conciencia poblacional, según se ha definido, existe una clara tendencia a que en sus explicaciones sobre la pobreza recurran a argumentos puramente socioeconómicos (alrededor de dos tercios). Prácticamente no hay malthusianos y la segunda orientación para explicar la pobreza tampoco recurre a argumentos económico-sociales ("otros", con $22.6 \%$ ). Por último, apenas poco más de una décima parte cree que los factores demográficos podrían tener algún papel que jugar en conjunción con los socioeconómicos ("eclécticos").

CUADRO 2

Explicaciones de la pobreza y conciencia poblacional de los maestros universitarios mexicanos de provincia (siete ciudades, 1987)

\begin{tabular}{lccrc}
\hline $\begin{array}{l}\text { Determinantes } \\
\text { de la pobreza }\end{array}$ & $\begin{array}{c}\text { Conciencia poblacional } \\
\text { "Conscientes" } \\
(n=902)\end{array}$ & $\begin{array}{c}\text { "Otros" } \\
(n=364)\end{array}$ & $\begin{array}{c}\text { Total } \\
(n=1266)\end{array}$ \\
\hline Eclécticos & 30.9 & 12.1 & 25.5 & $(\mathrm{n}=323)$ \\
Malthusianos & 10.3 & 1.6 & 7.8 & $(\mathrm{n}=99)$ \\
Radicales & 42.9 & 63.7 & 48.9 & $(\mathrm{n}=619)$ \\
Otros & 15.9 & 22.6 & 17.8 & $(\mathrm{n}=225)$ \\
Total & 100.0 & 100.0 & 100.0 & \\
& $(71.3)$ & $(28.8)$ & &
\end{tabular}

Chi cuadrado $=90.49382$, significativo al .0000

Fuente: Encuesta a maestros universitarios de provincia, Fondo de Población, Nu/U. Iberoamericana. 
Debe notarse que en el cuadro anterior dicotomizamos la "conciencia poblacional". Sin embargo, y dado que con ello reunimos opiniones que no son necesariamente idénticas, conviene ver hasta qué punto distintos tipos de conciencia afectan las explicaciones acerca de la pobreza. La información se presenta en el cuadro 3, donde se separó a los "alertas" de los "preocupados" (panel superior) y a los "optimistas" de los "otros".

Puede apreciarse que entre los que tienen alguna conciencia de la problemática poblacional hay una clara diferencia con respecto a la manera como explican la pobreza: mientras más de la mitad de los "alertas" otorgan alguna responsabilidad a los factores demográficos (predominando las explicaciones "eclécticas" por sobre las "malthusianas"), alrededor de $60 \%$ de los "preocupados" recurren a explicaciones únicamente socioeconómicas y nada más una ínfima minoría a factores sólo demográficos. Entre los "preocupados", por último, los "eclécticos" fueron sólo 15.8 por ciento.

Por otro lado, quienes no tienen una conciencia poblacional en el sentido en que la hemos definido -esto es, "optimistas" y "desapercibidos"- tienden a ser radicales, pero hay una fuerte diferencia de proporciones entre ellos (14.5 puntos más en el caso de los "radicales").

Además, y no menos importante, mientras alrededor de una cuarta parte de los "desapercibidos" tenían una visión equilibrada de los determinantes de la pobreza, menos de $10 \%$ de los "optimistas" dieron algún crédito a los factores demográficos. En ambos casos las posiciones "malthusianas" son irrelevantes.

En síntesis, al desagregar los "conscientes" quedó en evidencia que los de mayor nivel de conciencia poblacional, representados por los "alertas", recurrieron en mayor medida a explicaciones demográficas de la pobreza que los meramente "preocupados", mientras que, por otro lado, entre los que carecen de conciencia poblacional los que recurrieron a ese tipo de explicaciones fueron sólo una cuarta parte de los "desapercibidos".

Ahora bien, al tomar el conjunto de la información presentada, surgen claramente tres grandes agrupaciones en las formas de conciencia y la explicación de la pobreza que, en conjunto, comprenden a $84.8 \%$ de los maestros entrevistados:

1) Un primer grupo se compone de los que exhiben alguna conciencia poblacional y ofrecen una explicación de la pobreza en la que los aspectos demográficos tienen un papel (372 casos o $29.4 \%$ del total de entrevistados: véase la primera casilla del cuadro 2 , "conscientes eclécticos" y "conscientes malthusianos"). Dentro de este grupo, el núcleo está constituido por los "eclécticos", esto es, los que, teniendo alguna conciencia poblacional, atribuyen la po- 
CUADRO 3

Explicaciones de la pobreza y conciencia poblacional de los maestros universitarios mexicanos de provincia: desagregando entre "conscientes" y "otros" (siete ciudades, 1987)

\begin{tabular}{lcccc}
\hline $\begin{array}{l}\text { Determinantes } \\
\text { de la pobreza }\end{array}$ & \multicolumn{3}{c}{ Con "Conciencia poblacional" } \\
\cline { 2 - 5 } & $\begin{array}{c}\text { Alertas" } \\
(n=567)\end{array}$ & $\begin{array}{c}\text { "Preocupados" } \\
(n=335)\end{array}$ & \multicolumn{2}{c}{$\begin{array}{c}\text { Total } \\
(n=902)\end{array}$} \\
\hline Eclécticos & 39.9 & 15.8 & 30.9 & $(\mathrm{n}=279)$ \\
Malthusianos & 13.2 & 5.4 & 10.3 & $(\mathrm{n}=93)$ \\
Radicales & 14.3 & 60.3 & 42.9 & $(\mathrm{n}=387)$ \\
Otros & 14.3 & 18.5 & 15.9 & $(\mathrm{n}=143)$ \\
Total & $\mathbf{1 0 0 . 0}$ & 100.0 & 100.0 & \\
& $(62.9)$ & $(37.1)$ & &
\end{tabular}

Chi cuadrado $=91.88601$, significativo al .0000

\begin{tabular}{lccrl}
\hline & \multicolumn{3}{c}{ Sin "conciencia poblacional" } \\
\cline { 2 - 5 } $\begin{array}{l}\text { Determinantes } \\
\text { de la pobreza }\end{array}$ & $\begin{array}{c}\text { Optimistas" } \\
(n=276)\end{array}$ & $\begin{array}{c}\text { "Desapercib." } \\
(n=87)\end{array}$ & \multicolumn{2}{c}{$\begin{array}{c}\text { Total } \\
(n=363)\end{array}$} \\
\hline Eclécticos & 8.0 & 24.1 & $11.8 \quad(\mathbf{n}=43)$ \\
Malthusianos & 1.8 & 1.1 & $1.7 \quad(\mathrm{n}=6)$ \\
Radicales & 67.4 & 52.9 & $63.9 \quad(\mathrm{n}=232)$ \\
Otros & 22.8 & 21.8 & $22.6 \quad(\mathbf{n}=82)$ \\
Total & 100.0 & 100.0 & 100.0 & \\
& $(62.9)$ & $(37.1)$ & &
\end{tabular}

Chi cuadrado $=16.98076$, significativo al .0007

Fuente: Encuesta a maestros universitarios de provincia, Fondo de Población, $\mathrm{NU} / \mathrm{U}$. Iberoamericana.

breza tanto a factores demográficos como socioeconómicos (279 casos o $22.1 \%$ del total de casos).

2) Un segundo conjunto está representado por los que, teniendo alguna conciencia poblacional, creen sin embargo que la pobreza se explica por factores socioeconómicos y no por los demográficos ("alertas" y "preocupados" radicales: 387 casos o $30.6 \%$ del total: cuadro 3, panel superior).

3) Otro grupo de importancia está compuesto por quienes no ven exceso de población ni problema en su crecimiento y creen, o bien que la pobreza es causada por la concentración de la riqueza o bien por otros factores distintos de los poblacionales y socioeconómicos (314 casos, o 24.8\%, cuadro 2). El núcleo de este grupo está conformado por los "optimistas radicales" (186 casos o $14.7 \%$ del total de entrevistados). 
En suma, es claro que una cierta moderación de las opiniones respecto de las causas de la pobreza ("eclécticos") se vincula con la mayor conciencia poblacional, mientras que el radicalismo aparece asociado tanto a la conciencia como, sobre todo, al "optimismo" respecto de cuestiones poblacionales. Debe destacarse además que, en la explicación de la pobreza, en ninguno de estos tipos tuvo importancia el malthusianismo; en el mejor de los casos, alcanzó poco más de $13 \%$ entre los "alertas". Por tanto, si bien cierto "radicalismo" se vincula con el rechazo de los aspectos demográficos del crecimiento como problema, las posiciones no resultan tan polarizadas como podría haberse presumido.

\section{Crecimiento demográfico y desarrollo deseable}

A diferencia de lo que acontece con las vinculaciones entre crecimiento demográfico y pobreza, tratándose de los tipos de desarrollo deseable, las posibilidades de polarización de las opiniones disminuyen considerablemente. Ya se indicaron las razones que podrían jugar. Los costos de las opciones polares pueden ser enormes si se los asume desde una perspectiva estrecha: descansar básicamente en la incorporación de los avances tecnológicos podría tener graves consecuencias sobre el empleo, agudizando la disparidad; despreocuparse de ellos podría llevar a enormes esfuerzos humanos sin que se llegara al logro de la meta. Por otra parte, la experiencia histórica del desarrollo mexicano, donde ha predominado una ideología de claros rasgos populistas, lleva a que se asuma, de modo casi natural, la vía ecléctica. En el cuadro 4 se puede encontrar una cierta corroboración de lo anterior.

A pesar del amplio predominio de las opiniones en favor del desarrollo equilibrado, existen diferencias entre los que tienen alguna conciencia y los que no: es mayor la proporción de los primeros que optó por el equilibrio y algo menor la que dio otras respuestas. Las proporciones que enfatizaron el uso de la fuerza de trabajo o la tecnología fueron bajas y similares en su peso.

Conviene ahora ver si existen diferencias en las opiniones acerca del tipo de desarrollo deseable según el tipo particular de conciencia existente (cuadro 5).

De la lectura del cuadro queda en claro, primero, que es mayor la proporción global de los que prefieren el equilibrio en el desarrollo entre los que tienen alguna conciencia por comparación con los que no la tienen. Por otro lado, entre los primeros, son los "alertas" los más interesados en el equilibrio (71.4\% ver- 


\section{CUADRO 4}

Tipo de desarrollo deseable y conciencia poblacional de los maestros universitarios mexicanos de provincia (siete ciudades, 1987)

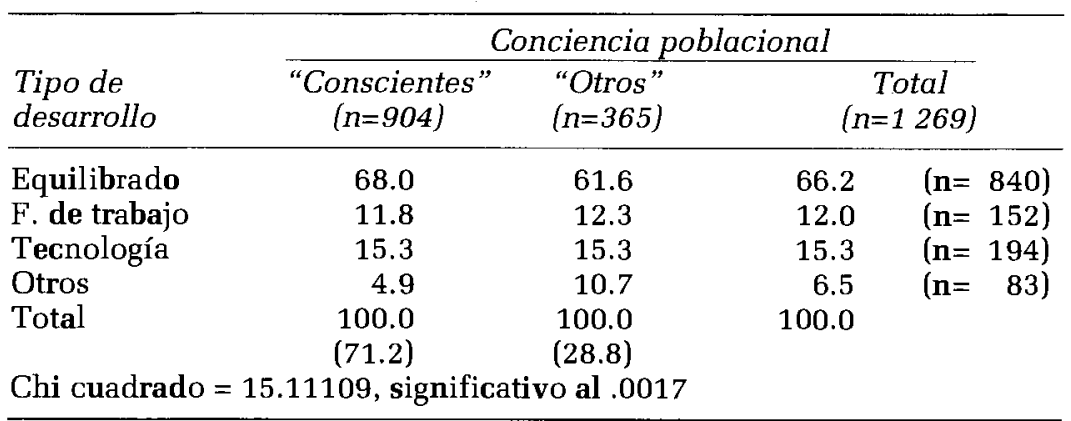

Fuente: Encuesta a maestros universitarios de provincia, Fondo de Población, NU/U. Iberoamericana.

\section{CUADRO 5}

Tipo de desarrollo deseable y conciencia poblacional de los maestros universitarios mexicanos de provincia: desagregando "conscientes" y "otros" (siete ciudades, 1987)

\begin{tabular}{|c|c|c|c|c|c|}
\hline \multirow{3}{*}{$\begin{array}{l}\text { Tipo de } \\
\text { desarrollo }\end{array}$} & \multicolumn{5}{|c|}{ Con "conciencia poblacional" } \\
\hline & \multirow{2}{*}{$\begin{array}{c}\text { "Alertas" } \\
(n=567)\end{array}$} & \multirow{2}{*}{$\begin{array}{c}\text { "Preocupados" } \\
(n=337) \\
62.3\end{array}$} & \multicolumn{3}{|c|}{$\begin{array}{c}\text { Total } \\
(n=904)\end{array}$} \\
\hline & & & 68.0 & $(\mathrm{n}=$ & 615) \\
\hline F. de trabajo & 10.6 & 13.9 & 11.8 & ( $\mathrm{n}=$ & 107) \\
\hline Tecnología & 13.8 & 17.8 & 15.3 & $(\mathrm{n}=$ & 138) \\
\hline Otros & 4.2 & 5.9 & 4.9 & $(n=$ & 44) \\
\hline Total & $\begin{array}{c}100.0 \\
(62.7)\end{array}$ & $\begin{array}{r}100.0 \\
(37.2)\end{array}$ & 100.0 & & \\
\hline
\end{tabular}

Chi cuadrado $=8.12865$, significativo al .0434

\begin{tabular}{|c|c|c|c|c|c|}
\hline \multirow{3}{*}{$\begin{array}{l}\text { Tipo de } \\
\text { desarrollo } \\
\text { Equilibrado }\end{array}$} & \multicolumn{5}{|c|}{ Sin "conciencia poblacional" } \\
\hline & \multirow{2}{*}{$\frac{\begin{array}{c}\text { Optimistas" } \\
(n=275)\end{array}}{60.4}$} & \multirow{2}{*}{$\frac{\begin{array}{c}\text { "Desapercib." } \\
(n=87)\end{array}}{65.5}$} & \multicolumn{3}{|c|}{$\begin{array}{c}\text { Total } \\
(n=362)\end{array}$} \\
\hline & & & 61.6 & (n) $=$ & 223) \\
\hline F. de trabajo & 12.4 & 12.6 & 12.4 & (n $=$ & 45) \\
\hline Tecnología & 15.4 & 13.8 & 15.2 & $(\mathbf{n}=$ & 55) \\
\hline Otros & 11.6 & 8.0 & 10.8 & $\mathrm{n}=$ & 39) \\
\hline Total & $\begin{array}{l}100.0 \\
(76.0)\end{array}$ & $\begin{array}{r}100.0 \\
(24.0)\end{array}$ & 100.0 & & \\
\hline
\end{tabular}

Chi cuadrado $=1.22772$, no significativo

Fuente: Encuesta a maestros universitarios de provincia, Fondo de Población, Nu/U. Iberoamericana. 
sus $62.3 \%$ de los "preocupados"). Y si bien entre los que no tienen preocupaciones sobre la significación de la población son los "otros" los que parecen más moderados, la diferencia respecto de los "optimistas" no es significativa.

\section{Desarrollo deseable, pobreza y conciencia poblacional}

Se ha dicho antes que es razonable suponer que existe alguna vinculación entre la conciencia poblacional y las explicaciones de la pobreza pero que, tratándose de las preferencias por el tipo de desarrollo deseable, las vinculaciones con la conciencia poblacional son más difíciles de establecer. Con todo, el análisis anterior mostró que, siendo general la preferencia por el equilibrio en el desarrollo, era mayor entre los que denominamos "alertas". En otras palabras, que son precisamente los más preocupados por el tamaño y el crecimiento de la población los que mayormente preferirían un desarrollo económico equilibrado.

A partir de lo anterior, nos interesó tener una idea un poco más profunda acerca de las vinculaciones entre desarrollo deseable, explicaciones de la pobreza y conciencia poblacional. Para ello, examinamos la vinculación entre la pobreza y el desarrollo deseable distinguiendo entre los que aducían algún factor demográfico en su explicación y los que no lo hacían, y controlamos por el tipo de conciencia poblacional. En el cuadro 6, ésta fue igualmente dicotomizada entre "conscientes" en general y "otros".

$41.2 \%$ de los que mostraron alguna preocupación por el tamaño o crecimiento de la población explicaron la pobreza dando alguna importancia a factores demográficos, en comparación con sólo $13.7 \%$ de los que no dieron importancia a las características de la población.

Por tanto, el peso de las explicaciones puramente socioeconómicas en la determinación de la pobreza fue mayor entre los últimos que entre los conscientes ( $86.3 \%$ y $58.8 \%$, respectivamente).

Por otra parte, al estudiar la parte superior del cuadro, resulta claro que los conscientes que incluyen un factor demográfico en la explicación de la pobreza son los más interesados en un desarrollo equilibrado $(72.8 \%$ en comparación con los conscientes de orientación socioeconómica en cuanto a la pobreza, que fueron poco menos de dos tercios de los entrevistados).

Si bien aparecen diferencias entre los que no tienen conciencia poblacional en relación con el peso que otorgan a lo demográfico para explicar la pobreza y en las proporciones en que se 
CUADRO 6

Tipo de desarrollo deseable, explicaciones de la pobreza y conciencia poblacional de los maestros universitarios mexicanos de provincia (siete ciudades, 1987)

\begin{tabular}{|c|c|c|c|c|}
\hline \multicolumn{5}{|c|}{ A: profesores con "conciencia poblacional" } \\
\hline \multirow[b]{2}{*}{ Tipo desarrollo } & \multicolumn{4}{|c|}{$\begin{array}{l}\text { La explicación } \\
\text { de la pobreza tiene un componente poblacional }\end{array}$} \\
\hline & $\begin{array}{c}\text { Sí } \\
(n=372)\end{array}$ & $\begin{array}{c}\text { No } \\
(n=532)\end{array}$ & $\begin{array}{c}\text { Total } \\
(n=904)\end{array}$ & \\
\hline Equilibrado & 72.8 & 64.7 & 68.0 & $(\mathbf{n}=615)$ \\
\hline F. de trabajo & 9.4 & 13.5 & 11.8 & $(\mathbf{n}=107)$ \\
\hline Tecnología & 15.3 & 15.2 & 15.3 & $(\mathrm{n}=138)$ \\
\hline Otros & 2.4 & 6.6 & 4.9 & $(\mathbf{n}=44)$ \\
\hline Total & 100.0 & $\begin{array}{r}100.0 \\
(58.8)\end{array}$ & 100.0 & \\
\hline \multicolumn{5}{|c|}{ Chi cuadrado $=13.08841$, significativo al .0044} \\
\hline \multicolumn{5}{|c|}{ B: profesores sin "conciencia poblacional" } \\
\hline & \multicolumn{4}{|c|}{$\begin{array}{l}\text { La explicación } \\
\text { de la pobreza tiene un componente poblacional }\end{array}$} \\
\hline Tipo desarrollo & $\begin{array}{c}S i ́ \\
(n=50)\end{array}$ & $\begin{array}{c}\text { No } \\
(n=315)\end{array}$ & $\begin{array}{c}\text { Total } \\
(n=365)\end{array}$ & \\
\hline Equilibrado & 70.0 & 60.3 & 61.6 & $(\mathrm{n}=225)$ \\
\hline F. de trabajo & 8.0 & 13.0 & 12.3 & $(\mathrm{n}=45)$ \\
\hline Tecnología & 14.0 & 15.6 & 15.3 & $(n=$ \\
\hline Otros & 8.0 & 11.1 & 10.7 & (n = \\
\hline Total & $\begin{array}{r}100.0 \\
(13.7)\end{array}$ & $\begin{array}{r}100.0 \\
(86.3)\end{array}$ & 100.0 & \\
\hline \multicolumn{5}{|c|}{ Chi cuadrado $=1.99576$, no significativo } \\
\hline
\end{tabular}

Fuente: Encuesta a maestros universitarios de provincia, Fondo de Población, Nu/U. Iberoamericana.

orientan al equilibrio en el desarrollo, éstas últimas no son significativas. Se notará, en efecto, que al desagregar los tipos de conciencia entre, por un lado, "alertas" y "preocupados" y, por otro, "optimistas" y "otros", se mantiene la misma estructura de relaciones (la información se presenta en los cuadros 7 y 8 , en el anexo).

En síntesis, esto quiere decir que: a) los alertas que incluyen un factor poblacional en la explicación de la pobreza son poco más de la mitad de la categoría y que, entre éstos, el interés por el desarrollo equilibrado es mayor que en el caso de los que explican la pobreza sin recurrir a factores demográficos (cuadro 7). 
Asimismo b), que las diferencias entre los meramente "preocupados" no son significativas en lo que se refiere a la vinculación entre explicación de la pobreza e interés en el equilibrio (cuadro 7), y lo mismo resulta en el caso de los carentes de conciencia (cuadro 8 ).

Puede concluirse, entonces, que la preocupación por un desarrollo equilibrado, siendo general, es más fuerte entre los conscientes de la problemática poblacional que, además, ven en ésta una determinante de la pobreza.

\section{Síntesis y conclusiones}

En este trabajo reseñamos brevemente los contenidos básicos de las discusiones que se han suscitado en torno a las relaciones entre crecimiento demográfico y desarrollo económico y sus interpretaciones. En particular, nos interesó indagar por la presencia que las posiciones históricamente en juego pudieran tener en la manera como los maestros universitarios de provincia evaluaban, en la segunda mitad de los años ochenta, el significado del tamaño y el crecimiento poblacional respecto del desarrollo y la pobreza.

También estudiamos las preferencias por el desarrollo deseable, circunscribiéndonos al énfasis dado a la incorporación de tecnología o al uso de la fuerza de trabajo disponible.

El análisis presentado sugiere que ha existido un cierto impacto de las polémicas sobre población y desarrollo en las opiniones que los profesores entrevistados enunciaron sobre los aspectos específicos examinados en este trabajo. Éste se expresaría, por la vía negativa, en la presencia de un importante contingente de maestros de posiciones "radicales", que no conceden importancia ni al crecimiento poblacional ni a sus posibles efectos sobre el desarrollo o la explicación de la pobreza. Y, por la vía positiva, en cierta variedad en las orientaciones de los maestros universitarios, que no obstante mayoritariamente tienden a atribuir algún peso a los factores demográficos. En efecto, aun entre quienes no atribuyen importancia a los factores demográficos en la explicación de la pobreza, se ha abierto camino la idea de que los problemas del tamaño y el crecimiento de la población constituyen o pueden constituir un problema para el futuro del país (éstos podrían referirse, por ejemplo, a cuestiones relativas al grado en que las oportunidades que ha abierto el desarrollo podrían verse afectadas, al ritmo de crecimiento del empleo, etcétera).

Por otra parte, los resultados muestran que, en lo que se refiere a las orientaciones respecto del desarrollo que se estima deseable, 
tiende a haber coincidencia entre los maestros, en general con independencia de las orientaciones que tengan en relación con el significado de la población y la explicación de la pobreza: la mayor parte preferiría un desarrollo que combinara la incorporación de tecnología con el uso de la fuerza de trabajo disponible.

En nuestro análisis distinguimos tres tipos básicos de conciencia poblacional en referencia a las explicaciones de la pobreza (véase el apartado sobre "crecimiento demográfico y pobreza"). Combinando esos tipos con las orientaciones respecto del tipo de desarrollo deseable, surgen algunos perfiles básicos de interés. Podría conjeturarse la estructura elemental de los argumentos subyacentes (nos referiremos a los núcleos fundamentales de cada agrupación).

\section{Conscientes eclécticos}

a) "El país tiene (o puede tener) un exceso de población y su crecimiento representa un obstáculo para el desarrollo futuro; por otra parte,

b) los factores demográficos, por sí mismos o en conjunción con los procesos que llevan a la concentración de la riqueza, tienen un papel que jugar en la explicación de la pobreza existente;

c) en consecuencia, lo razonable sería buscar la promoción del desarrollo aumentando la productividad y generando a la vez nuevas fuentes de empleo que permitieran usar la fuerza de trabajo disponible (excedente). De esta manera, tal vez se podría adelantar en la tarea del desarrollo sin generar una desocupación excesiva, resultante de la sobrepoblación, actual o potencial, existente”.

\section{Conscientes radicales}

Esta variante reconocería en el crecimiento de la población si no un problema actual, al menos una amenaza potencial para el futuro. Por otro lado, prescindiría de los factores demográficos en la explicación de la pobreza existente. Ésta se explicaría más bien por el proceso de concentración de la riqueza en pocas manos -aunque podría aceptar que en el futuro el crecimiento poblacional no controlado pudiese llegar a tener también un papel en la agudización o, aun, en la generación de la pobreza. En todo caso, esta posición coincidiría con las otras en que el tipo de desarrollo deseable debería combinar la incorporación de avances tecnológicos con el empleo de la fuerza de trabajo excedente. 
Optimistas radicales

Finalmente, la visión de éstos podría expresarse aproximadamente como sigue:

a) "La población de México no es excesiva y el país puede aceptar, sin problemas, su crecimiento; éste tampoco parece ser un problema para el futuro (por lo menos, próximo).

b) Atribuir la pobreza a factores demográficos es desconocer, consciente o inconscientemente, el papel determinante que tiene el estilo de desarrollo, que ha llevado a una creciente concentración de la riqueza y al mantenimiento o aumento de la pobreza, independientemente de los factores poblacionales.

c) Como quiera que sea, lo deseable sería un tipo de desarrollo que, incorporando los avances de la tecnología, fuese también capaz de emplear la fuerza de trabajo (excedente) de que dispone el país". 


\section{Anexo}

CUADRO 7

Tipo de desarrollo deseable, según explicaciones de la pobreza: maestros con "conciencia poblacional": desagregando entre "alertas" y "preocupados" (siete ciudades, 1987)

\begin{tabular}{|c|c|c|c|c|}
\hline \multicolumn{5}{|c|}{ "Alertas" } \\
\hline \multirow[b]{2}{*}{$\begin{array}{l}\text { Tipo de } \\
\text { desarrollo }\end{array}$} & \multicolumn{4}{|c|}{$\begin{array}{c}\text { La explicación } \\
\text { de la pobreza tiene un componente poblacional: }\end{array}$} \\
\hline & $\begin{array}{c}S i \\
(n=301)\end{array}$ & $\begin{array}{c}N o \\
(n=266)\end{array}$ & $\begin{array}{c}\text { Total } \\
(n=567)\end{array}$ & \\
\hline Equilibrado & 74.8 & 67.7 & 71.4 & $(n=405)$ \\
\hline F. de trabajo & 9.0 & 12.4 & 10.6 & $(\mathrm{n}=60)$ \\
\hline Tecnología & 14.0 & 13.5 & 13.8 & $(n=$ \\
\hline Otros & 2.3 & 6.4 & 4.2 & $(\mathbf{n}=$ \\
\hline Total & $\begin{array}{r}100.0 \\
(53.1)\end{array}$ & $\begin{array}{r}100.0 \\
(46.9)\end{array}$ & 100.0 & \\
\hline
\end{tabular}

Chi cuadrado $=8.09857$, significativo al .0440

\begin{tabular}{|c|c|c|c|c|}
\hline \multicolumn{5}{|c|}{ "Preocupados" } \\
\hline \multirow[b]{2}{*}{$\begin{array}{l}\text { Tipo de } \\
\text { desarrollo }\end{array}$} & \multicolumn{4}{|c|}{$\begin{array}{l}\text { La explicación } \\
\text { de la pobreza tiene un componente poblacional: }\end{array}$} \\
\hline & $\begin{array}{c}S i \\
(n=71)\end{array}$ & $\begin{array}{c}\text { No } \\
(n=266)\end{array}$ & $\begin{array}{c}\text { Total } \\
(n=337)\end{array}$ & \\
\hline Equilibrado & 64.8 & 61.7 & 62.3 & $(\mathrm{n}=210)$ \\
\hline F. de trabajo & 11.3 & 14.7 & 13.9 & $(\mathrm{n}=47)$ \\
\hline Tecnología & 21.1 & 16.9 & 17.8 & $(\mathrm{n}=60)$ \\
\hline Otros & 2.8 & 6.8 & 5.9 & $(\mathrm{n}=20)$ \\
\hline Total & 100.0 & 100.0 & 100.0 & \\
\hline
\end{tabular}

Chi cuadrado $=2.58237$, no significativo

Fuente: Encuesta a maestros universitarios de provincia, Fondo de Población, $\mathrm{Nu} / \mathrm{U}$. Iberoamericana. 


\section{Anexo}

\section{CUADRO 8}

Tipo de desarrollo deseable, según explicaciones de la pobreza: maestros sin "conciencia poblacional": desagregando entre "optimistas" y "desapercibidos" (siete ciudades, 1987)

\begin{tabular}{lcccc}
\hline \multicolumn{4}{c}{ “Optimistas” } \\
\hline \multicolumn{4}{c}{$\begin{array}{c}\text { La explicación } \\
\text { de la pobreza tiene un componente poblacional: }\end{array}$} \\
\cline { 2 - 5 } Tipo de & Sí & No & Total \\
desarrollo & $(n=27)$ & $(n=248)$ & $(n=275)$ & \\
\hline Equilibrado & 59.3 & 60.5 & 60.4 & $(\mathbf{n}=166)$ \\
F. de trabajo & 11.1 & 12.5 & 12.4 & $(\mathrm{n}=34)$ \\
Tecnología & 22.2 & 14.9 & 15.6 & $(\mathrm{n}=43)$ \\
Otros & 7.4 & 12.1 & 11.6 & $(\mathrm{n}=32)$ \\
Total & 100.0 & 100.0 & 100.0 & \\
& $(9.8)$ & $(90.2)$ & &
\end{tabular}

Chi cuadrado $=1.33467$, no significativo

\begin{tabular}{|c|c|c|c|c|c|}
\hline \multicolumn{6}{|c|}{ "Desapercibidos" } \\
\hline \multirow[b]{2}{*}{$\begin{array}{l}\text { Tipo de } \\
\text { desarrollo }\end{array}$} & \multicolumn{5}{|c|}{$\begin{array}{l}\text { La explicación } \\
\text { de la pobreza tiene un componente poblacional: }\end{array}$} \\
\hline & $\begin{array}{c}S i ́ \\
(n=22)\end{array}$ & $\begin{array}{c}N o \\
(\mathrm{n}=65)\end{array}$ & $\begin{array}{c}\text { Total } \\
(\mathrm{n}=87)\end{array}$ & & \\
\hline Equilibrado & 81.8 & 60.0 & 65.5 & $(\mathbf{n}=$ & 57) \\
\hline F. de trabajo & 4.5 & 15.4 & 12.6 & $(\mathbf{n}=$ & 11) \\
\hline Tecnología & 4.5 & 16.9 & 13.8 & $(n=$ & 12) \\
\hline Otros & 9.1 & 7.7 & 8.0 & $(n=$ & 7) \\
\hline Total & 100.0 & $\begin{array}{r}100.0 \\
(74.7)\end{array}$ & 100.0 & & \\
\hline
\end{tabular}

Chi cuadrado $=4.58725$, no significativo

Fuente: Encuesta a maestros universitarios de provincia, Fondo de Población, NU/U. Iberoamericana. 


\section{Bibliografía}

Alba, Francisco y Joseph Potter (1986), "Population and Development in Mexico since 1940: An Interpretation", en Population and Development Review, vol. 12, núm. 1, pp. 47-75.

Anker, Richard y Ghazi M. Farooq (1978), "Población y desarrollo socioeconómico: una nueva perspectiva”, en Revista Internacional del Trabajo, vol. 97, núm. 2, pp. 215-228.

Argüello, Omar (1979), "Pobreza, población y desarrollo", en Notas de Población, Santiago, Chile, año vII, núm. 20, pp. 73-112.

Asociación Nacional de Universidades e Instituciones de Educación Superior (ANuEs) (1986), Anuario Estadístico 1986. Licenciatura, México.

Astorga Almanza, Luis A. (1987), Genealogía y crítica de la "política de población" en México, México, Instituto de Investigaciones Sociales, Universidad Nacional Autónoma de México (Cuadernos de Investigación Social, 16).

Benítez Zenteno, Raúl (1965), “La población rural y urbana en México”, en Joseph Kahl (comp.), La industrialización en América Latina, México, Fondo de Cultura Económica, pp. 40-53.

- (1989), "Presentación", en Demos, núm. 1, pp. 2-3.

Cabrera, Gustavo (1984), "Notas sobre integración de las políticas de población. Investigación ¿para qué, para quién?”, en Memorias del Congreso Latinoamericano de Población y Desarrollo, México, unam/El Colegio de México/PISPaL, vol. 1, pp. 437-444.

Carrillo Flores, Antonio (coord.) (1974), Diálogos sobre población, México, El Colegio de México.

CEPAL (1983), "Situación demográfica de América Latina evaluada en 1983: estimaciones para 1960-1980 y proyecciones para 1980-2025”, en Notas de población, Santiago, Chile, año xı, núm. 33, pp. 9-65.

Coale, A. J. (1982), “Crecimiento de la población y desarrollo económico: el caso de México”, en Alejandro Mina (comp.), Lecturas sobre temas demográficos, México, El Colegio de México, pp. 255-270.

y E. M. Hoover (1958), Population Growth and Economic Development in Low-Income Countries, Princeton, Princeton University Press.

Cortés, Fernando (1982), "Tamaño de muestra y análisis de asociación", en Revista Mexicana de Sociología, núm. 4, pp. 1381-1411.

- y Rosa María Rubalcava (1991), Autoexplotación forzada y equidad por empobrecimiento, México, El Colegio de México.

Eversley, D. E. C. (1959), Social Theories of Fertility and the Malthusian Debate, Oxford, Clarendon Press.

Fucaraccio, Ángel (1979), "Población, fuerza de trabajo y desarrollo económico en América Latina”, en Víctor L. Urquidi y José B. Morelos (comps .), Población y desarrollo en América Latina, México, El Colegio de México, pp. 30-49.

González, Gerardo (1984) “Utilización del conocimiento en materia de población en las acciones para el desarrollo", en Memorias del Congreso Latinoamericano de Población y Desarrollo, México, unam/El Colegio de México/pispal, vol, 1, pp. 393-411. 
Leñero, Luis (1974), "Situación y perspectivas demográficas de México", en Antonio Carrillo Flores (coord.), Diálogos sobre población, México, El Colegio de México, pp. 147-186.

Mason, Andrew (1987), "Saving, Economic Growth, and Demographic Change", en Population and Development Review, vol. 14, núm. 1, pp. 113-144.

Meadows, Donella A., Dennis L. Meadows, Jorgen Randers y William W. Behrens III (1972), Los límites del crecimiento, México, Fondo de Cultura Económica.

Mesarovic, M. y E. Pestel (1974), La humanidad en la encrucijada, México, Fondo de Cultura Económica.

Miró, Carmen y Joseph E. Potter (1980), Población y desarrollo. Estado del conocimiento y prioridades de investigación, México, El Colegio de México.

Muñiz, Patricia (1988), "Antecedentes sociales, estudios y percepciones de la problemática poblacional de los profesores universitarios. Un análisis de 4 ciudades de la República mexicana", en Universidad, población y desarrollo, México, Universidad Iberoamericana/FNUAP, noviembre.

(1989), "¿Conocen los docentes universitarios las políticas de población? Un estudio en 6 ciudades", en Umbral XXI, México, núm. 1, pp. 32-36.

Petch, Waldomiro (1979), "Dinámica demográfica y desarrollo económico y social en América Latina", en Víctor L. Urquidi y José B. Morelos (comps.), Población y desarrollo en América Latina, México, El Colegio de México, pp. 11-29.

Preston, Samuel H. (1987), "Are the Economic Consequences of Population Growth a Sound Basis for Population Policy?", en Population Bulletin of the United Nations, núms. 23-24, pp. 1-10.

Rodgers, Gerry (1983), "Crecimiento de la población, disparidad y pobreza", en Revista Internacional del Trabajo, vol. 102, núm. 3, pp. 363-383.

Rodríguez, Daniel y Ricardo Yocelevzky (1986), Política y población en América Latina. Revisión de los aportes del Pispal, México, PISPAL/ El Colegio de México.

United Nations (1989a), Case Studies in Population Policy: Mexico, Nueva York, Department of International Economic and Social Affairs (Population Policy Paper, 21).

United Nations (1989b), World Population at the Turn of the Century, Nueva York, Department of International Economic and Social Affairs (Population Studies, 111).

United Nations Secretariat (1987), "The World Population Plan of Action and the Regional Commissions", en Population Bulletin of the United Nations, núms. 23-24, pp. 76-82.

Urquidi, Víctor L. y José B. Morelos (comps.) (1979), Población y desarrollo en América Latina, México, El Colegio de México. 